\title{
The possibilities and challenges of "Rehabilitation 2030: a call for action" by the World Health Organization: a unique opportunity not to be missed
}

\author{
Stefano NEGRINI 1, $2 *$ \\ ${ }^{1}$ Clinical and Experimental Sciences Department, University of Brescia, Brescia, Italy; ${ }^{2}$ IRCCS Fondazione Don Gnocchi, Milan, Italy \\ *Corresponding author: Stefano Negrini, Clinical and Experimental Sciences Department, University of Brescia, Brescia, Italy. E-mail: stefano.negrini@, \\ unibs.it
}

66 R ehabilitation 2030: a call for action" by the World Health Organization (WHO) ${ }^{1}$ can really constitute a huge advance for persons with disabilities (PWD) and all patients requiring rehabilitation to reduce their disability and improve their functioning. We decided to conclude this Special Section of the European Journal of Physical and Rehabilitation Medicine by starting a debate through this, let's say, "instant comment": the discussion has to be started in our clinical world, and these first thoughts are meant to be somehow provocative rather than definitive. We plan to produce more papers on the topic in the near future, involving all the most important actors, to help push forward the action called for by the WHO. These few paragraphs, coming from our specific position as a Physical and Rehabilitation Medicine (PRM) journal based in Europe and mostly in High Income Countries (HIC), are meant to stimulate the debate, and to better focus on some of the points to be considered.

\section{A cultural change}

The WHO clearly underlined the almost exclusive focus of national health systems today on curative and preventive strategies, largely reducing the space of rehabilitative and palliative strategies. ${ }^{1}$ In the face of the future challenges of all societies, from HIC to Low-Middle Income Countries (LMIC), aging of the population and the continuously improving curative possibilities increase the numbers of PWD, and of people requiring PRM. Whatever the possible explanation, this is a fact.

A problem probably not sufficiently focused on is that the prevalence of curative and preventive strategies does not come from conscious political or scientific choices, but mainly from a cultural and social perspective. Some examples will suffice:

- all government health agendas struggle with the needs of addressing an aging population, and the need to move from hospitals to outpatient treatment; we know that:

- PRM is a good answer to these needs;2,3

- robust PRM research is required to increase effective actions; 4

- PRM research is steadily growing; 5

nevertheless, PRM is nowadays undermined by the totally disease-oriented way of funding research by these same governments; ${ }^{6}$

- physicians' education is totally focused on the biomedical approach (morbidity and mortality), and (almost) totally ignores the rehabilitative strategy (functioning): in almost all countries undergraduate teaching of rehabilitation is reduced to a few hours and one single, little exam in a time frame of 5-6 years of studies to become a Doctor (MD); very often medical students are not even introduced to ICF; as a result, their knowledge of PRM is poor; 7 


\section{COPYRIGHT $^{(} 2017$ EDIZIONI MINERVA MEDICA}

- widespread ignorance of what PRM is, required to develop initiatives like the European White Book on $\mathrm{PRM},{ }^{2,3}$ or specific actions by scientific societies as in the USA (www.pmrismorethan.org; http://www.aapmr. org/about-physiatry/about-physical-medicine-rehabilitation);

- PRM value is underestimated in health settings, in political choices, as well as by the general public, since PRM does not deal with acute diseases or life threatening disorders.

These examples clearly point attention to a generalized cultural attitude that is obviously difficult to change. If we want to move toward "Rehabilitation 2030", we have to look at the situation in this wider perspective. WHO's call for action is a call for cultural change. A real challenge, but if we do not recognize it as such, there will be a very high probability of failure. Health systems will always be driven by political choices, patients' requirements, and money flow: today they concentrate almost exclusively on new treatments (for cancer, heart disease, hypertension, neurological disorders...) or disease prevention/diagnosis/etiology and not enough on rehabilitation, functioning, disability and quality of life improvement.

\section{Disentangle rehabilitation from disability as a health strategy}

This is somehow a novel concept of paramount importance that it has been raised by the WHO. Even in the world of PRM (which does not exactly overlap with rehabilitation, even if PRM is the medical specialty that deals with rehabilitation as a whole) the distinction between rehabilitation and disability, at least at a conceptual level, is not always completely clear. As an example, we can look at some definitions of rehabilitation and PRM. Until now, from the perspective of the WHO, the "Rehabilitation of people with disabilities is a process aimed at enabling them to reach and maintain their optimal physical, sensory, intellectual, psychological and social functional levels. Rehabilitation provides disabled people with the tools they need to attain independence and self-determination". ${ }^{8}$ According to the PRM Section of the European Union of Medical Specialists (UEMS), PRM is "an independent medical specialty concerned with the promotion of physical and cognitive functioning, activities (including behavior), participation (including quality of life) and modifying personal and environmental factors. It is thus responsible for the prevention, diagnosis, treatments and rehabilitation management of people with disabling medical conditions and comorbidity across all ages".2, 3 Finally, according to the American Board of Physical Medicine and Rehabilitation, some other concepts beyond disability are also introduced. PRM is defined as "a medical specialty concerned with diagnosis, evaluation, and management of persons of all ages with physical and/or cognitive impairment and disability." 9

When we look at PRM from this perspective of disentangling the two concepts of disability and rehabilitation, in our view some points should be considered such as:

- PWDs are the strength of PRM, since rehabilitation is their reference health strategy, even though they are not the only patients that PRM considers; PRM has to do with activity limitation but also with impairments; moreover, while many PRM patients end up with a disability, some recover completely;

- after a thorough initial rehabilitation process, PWDs can require PRM repeatedly, particularly in progressive diseases, but many need only maintenance; this is the last part of the rehabilitation process, ${ }^{2,3}$ but in most countries it is proposed today through the socalled "adapted physical activity", and not PRM:10 moreover, when the situation is clinically stable, there is a need for inclusive approaches by society that are more appropriate than direct medical PRM (e.g. removal of architectural barriers, or action by legislators).

The concept of disability is of the utmost importance for PRM whose point of reference is in fact the International Classification of Functioning, Disability and Health (ICF); ${ }^{11}$ nevertheless, rehabilitation is conceptually different from disability and should be considered per se. This is what the WHO has proposed with "Rehabilitation 2030".

\section{Scientific societies: the need for a team effort}

This WHO initiative should receive an appropriate answer from the international Scientific Societies. Their role is to cultivate science in their respective fields, in relation to their specific professional interests. Since there are so many professionals in rehabilitation, the result is that there are too many views and different ap- 


\section{COPYRIGHT ${ }^{(} 2017$ EDIZIONI MINERVA MEDICA}

proaches to make them really effective in the general interest. In a sense, having many perspectives and professionals can be the strong point of rehabilitation, but it is also its weakness if there is no unity.

As we know very well in everyday clinical work, if the team works well together, results are multiplied and made stronger; if the team does not function, results are even worse than those of a single professional treating the patient: confusion reigns, and the patient does not understand what to do. The same happens with respect to the outside world. Having different voices and professionals involved could be a real strong point if the different scientific societies expressing the interests and views of different professionals have the strength to work together; divisions should not prevail, and the rehabilitative strategy should be promoted setting up and making function an umbrella organization (best option) or an alliance (probably less effective) to collect and strengthen the shared needs of the rehabilitation world.

At this stage, given this unique opportunity, there should be no more room for jealousy, diffidence, fear of prevarication or whatever. The WHO is calling, and the rehabilitation world has to answer properly!

\section{Rehabilitation physicians are needed to make a change (also by other rehabilitation professionals)}

Beyond any other consideration, it is a fact that MDs have the most powerful voice in health services and that they manage health strategies providing health care. In this respect, having MDs as part of the rehabilitation team is a strength and should be in the interest of the whole team: it can be a major way for increasing the undervalued importance of rehabilitation among other health strategies. The low prevalence of PRM physicians all over the world as opposed to the much higher numbers of rehabilitation professionals 12,13 seems to drive political attention in other directions. And this is a problem for everybody in rehabilitation, whichever professional group is considered.

If we want to make a change in our approach to a health strategy, MDs are needed: if the change is related to rehabilitation, MDs specializing in rehabilitation (PRM) are needed. They can speak out, be heard, understood and recognized by all other MDs. Even if in a narrow minded approach this could be regarded as a paradox, rehabilitation professionals should strive to have more PRM specialists in their own interest. While the importance of PRM specialists might not be fully understood by many politicians and administrators, it should be by rehabilitation professionals.

On another note, in a more political and administrative view, it is proven that a comprehensive team approach to rehabilitation reduces costs, and there is no team without adequate management. ${ }^{14-16}$ This has been defined by the European Union as a medical responsibility:2,3 as such, it must be in the hands of an MD. ${ }^{17}$

Finally, we have to point out that, while in all European Union Countries but one there is a PRM medical specialty, 2,3 in some countries there are no University professorships; in almost all cases, there are far fewer PRM professors than in most of the other medical specialties. It is difficult to promote rehabilitation as a health strategy in the face of this situation.

\section{Research: we need appropriate and stronger evidence, with increased funding}

The role of scientific journals is to increase evidence in our field. One of the problems with evidence in rehabilitation is the difficulty of producing a meaningful one. Nevertheless, it is now recognized that evidence in rehabilitation (but not only) is not based only on Randomized Controlled Trials, but also on different designs, able to overcome the limits of RCTs in rehabilitation: 18,19 it is not by chance that this is one of the tasks of Cochrane Rehabilitation, ${ }^{20,21}$ with the final aim of decreasing the diffidence toward research in this area and to increase the possibility of producing valid evidence. This must go with strengthening the role of researchers in Rehabilitation, an increase in their number, and also an improvement in the quality of their work, so to produce more and more really meaningful evidence. If we really want to respond to the challenge of the WHO we definitively need evidence on which to base political decisions. Moreover, it is important that politicians channel money into an area of research that is still neglected because it is difficult and considered less rewarding. 5,6

\section{High income countries require changes as much as low middle income countries}

Obviously, the WHO has a crucial role to play in the Rehabilitation 2030 call for action: it started it, and it 


\section{COPYRIGHT $^{(} 2017$ EDIZIONI MINERVA MEDICA}

will lead it. Nevertheless, in Geneva it has been stated that the highest mandate is to focus on LMIC. We would like to argue about this statement. In fact, it is true that unmet needs are greater in LMIC; and sometimes it is paradoxically easier to do a very good job starting from a very low level. But the reality is that rehabilitation is a great unaddressed need in HIC too. This need is general, and facing it in the complex and multifaceted $\mathrm{HIC}$ can be even more challenging than in LMIC. These challenges must be met too, and it cannot be ignored that HIC can be facilitators, but also barriers to changes in LMIC, and not only in economic terms, but also as cultural references.

\section{Conclusions}

We can look at what happened in Geneva from various perspectives, but there was for sure and for all stakeholders' representatives that participated in the meeting a single common denominator: enthusiasm and hope for a brighter future for rehabilitation as a health strategy. The conclusion is that something has been started by the WHO, and that now the baton passes to the stakeholders: there is a great and unique responsibility on our shoulders to move forward, pushing ourselves with the WHO over the obstacles, to really make a difference for our patients, and our societies. This must be done now: there will not be other opportunities like this for years. But in the end, what is the rehabilitation world about, if not about overcoming barriers in the way to a better life?

\section{References}

1. World Health Organization. Rehabilitation 2030: a call for action: Meeting report [Internet]. WHO; 2017. Available from: http://www. who.int/disabilities/care/rehab-2030/en/ [cited 2017, Apr 5].

2. Section of Physical and Rehabilitation Medicine Union Européenne des Médecins Spécialistes (UEMS), European Board of Physical and Rehabilitation Medicine, Académie Européenne de Médecine de Réadaptation, European Society for Physical and Rehabilitation Medi- cine. White book on physical and rehabilitation medicine in Europe. Eur Medicophysica 2006;42:292-332.

3. [No authors listed]. White book on Physical and Rehabilitation Medicine in Europe. J Rehabil Med 2007;(45 Suppl):6-47.

4. Grimby G. Aspects on the research fields "from cell to society", clinical trials and research methodology, and report on impact. J Rehabil Med 2011;43:757-60.

5. Negrini S. Steady growth seen for research in physical and rehabilitation medicine: where our specialty is now and where we are going. Eur J Phys Rehabil Med 2012;48:543-8.

6. Negrini S, Padua L, Kiekens C, Michail X, Boldrini P. Current research funding methods dumb down health care and rehabilitation for disabled people and aging population: a call for a change. Eur J Phys Rehabil Med 2014;50:601-8.

7. Tederko P, Krasuski M, Denes Z, Moslavac S, Likarevic I. What medical doctors and medical students know about physical medicine and rehabilitation: a survey from Central Europe. Eur J Phys Rehabil Med 2016;52:597-605.

8. WHO | Rehabilitation; [Internet]. Available from: http://www.who. int/topics/rehabilitation/en/ [cited 2016, Aug 22].

9. The American Board of Physical Medicine and Rehabilitation [Internet]. Available from: https://www.abpmr.org/consumers/pmr_definition.html [cited 2016, Aug 22].

10. Italian Health Ministry. Rehabilitation national plan: an Italian act. Eur J Phys Rehabil Med 2011;47:621-38.

11. World Health Organization. WHO | International Classification of Functioning, Disability and Health (ICF); [Internet]. Available from: http://www.who.int/classifications/icf/en/ [cited 2014, Aug 19].

12. World Health Organization. The need to scale up rehabilitation; 2017 [Internet]. Available from: http://www.who.int/disabilities/care/ NeedToScaleUpRehab.pdf?ua=1 [cited 2017, Apr 4].

13. Gimigliano F, Negrini S. The World Health Organization "Rehabilitation 2030: a call for action". Eur J Phys Rehabil Med 2017;53:155-68.

14. Neumann V, Gutenbrunner C, Fialka-Moser V, Christodoulou N, Varela E, Giustini A, et al. Interdisciplinary team working in physical and rehabilitation medicine. J Rehabil Med 2010;42:4-8.

15. Körner M, Bütof S, Müller C, Zimmermann L, Becker S, Bengel J. Interprofessional teamwork and team interventions in chronic care: $\mathrm{A}$ systematic review. J Interprof Care 2016;30:15-28.

16. Momsen AM, Rasmussen JO, Nielsen CV, Iversen MD, Lund H. Multidisciplinary team care in rehabilitation: an overview of reviews. J Rehabil Med 2012:44:901-12.

17. Smits SJ, Bowden D, Falconer JA, Strasser DC. Improving medical leadership and teamwork: an iterative process. Leadersh Health Serv 2014;27:299-315

18. Malmivaara A. Benchmarking Controlled Trial--a novel concept covering all observational effectiveness studies. Ann Med 2015;47:33240.

19. Malmivaara A. Assessing validity of observational intervention studies - the Benchmarking Controlled Trials. Ann Med 2016;48:440-3.

20. Kiekens C, Negrini S, Thomson D, Frontera W. Cochrane Physical and Rehabilitation Medicine: Current State of Development and Next Steps. Am J Phys Med Rehabil Assoc Acad Physiatr 2016;95:235-8.

21. Negrini S, Kiekens C, Levack W, Grubisic F, Gimigliano F, Ilieva E, et al. Cochrane physical and rehabilitation medicine: a new field to bridge between best evidence and the specific needs of our field of competence. Eur J Phys Rehabil Med 2016;52:417-8.

Conflicts of interest.-The authors certify that there is no conflict of interest with any financial organization regarding the material discussed in the manuscript. (Cite this article as: Negrini S. The possibilities and challenges of "Rehabilitation 2030: a call for action" by the World Health Organization: a unique opportunity not to be missed. Eur J Phys Rehabil Med 2017;53:169-72. DOI: 10.23736/S1973-9087.17.04766-9) 